\title{
Impact of the strong electromagnetic field on the QCD effective potential for homogeneous Abelian gluon field configurations
}

\author{
Bogdan V. Galilo, Sergei N. Nedelko* \\ Bogoliubov Laboratory of Theoretical Physics, JINR, 141980 Dubna, Russia, \\ and Department of Theoretical Physcis, Dubna International University, 141980 Dubna, Russia
}

\begin{abstract}
The one-loop quark contribution to the QCD effective potential for the homogeneous Abelian gluon field in the presence of external strong electromagnetic field is evaluated. The structure of extrema of the potential as a function of the angles between chromoelectric, chromomagnetic and electromagnetic fields is analyzed. In this setup, the electromagnetic field is considered as an external one while the gluon field represents domain structured nonperturbative gluon configurations related to the QCD vacuum in the confinement phase. Two particularly interesting gluon configurations, (anti-)self-dual and crossed orthogonal chromomagnetic and chromoelectric fields, are discussed specifically. Within this simplified framework it is shown that the strong electromagnetic fields can play a catalysing role for a deconfinement transition. At the qualitative level, the present consideration can be seen as a highly simplified study of an impact of the electromagnetic fields generated in relativistic heavy ion collisions on the strongly interacting hadronic matter.

PACS numbers: $12.38 . \mathrm{Aw}, 12.38 . \mathrm{Lg}, 14.70 . \mathrm{Dj}$
\end{abstract}

\section{INTRODUCTION}

The purpose of this paper is to study a potential influence of the strong electromagnetic fields, $e H \simeq \Lambda_{\mathrm{Q} C D}^{2}$, on the QCD vacuum structure. Electromagnetic fields with the strength of this order can emerge in relativistic heavy ion collisions. Before proceeding, we have to decode our understanding of the stock phrase "QCD vacuum structure". In pure gluodynamics a physical vacuum can be characterized, first of all, by two invariants composed of gauge field: scalar gluon condensate $\left\langle g^{2} F^{2}\right\rangle$ and pseudoscalar condensate $\left\langle g^{2} F \tilde{F}\right\rangle$ (for instance see discussion in [1]). Since parity is not broken in strong interactions, the pseudoscalar condensate must be zero. Significance of the composite field $g^{2} F \tilde{F}$ becomes manifest in terms of topological susceptibility. In QCD with quarks another condensate $\langle m \bar{\psi} \psi\rangle$ comes into consideration. Identification of gauge field configurations which are carriers of condensates and the method of their incorporation into the formalism of quantum field theory can be seen as the most fundamental step towards understanding the mechanisms of confinement, chiral symmetry breaking and hadronization in QCD. This statement can be perceived as a kind of platitude since implicitly this step has to be assumed in all approaches dealing with configurations like center vortices, monopoles, instantons, etc. However, the feeling of having just a commonplace here relaxes if one identifies explicitly the point in the formalism where relevant condensates can be allowed or denied to be nonzero. As has been amphasized in a recent paper 2], this point can be recognised in the choice of a functional space of the gauge fields to be integrated over in the QCD functional integral. In the Euclidean functional integral approach to quantization of the pure YM theory one starts with a symbol

$$
Z=N \int_{\mathcal{F}} D A \exp \{-S[A]\},
$$

where the functional space $\mathcal{F}$ of fields is subject to certain conditions, which can disable, in particular, the gluon condensate (requirement of finite classical action $S[A]$, for instance) or enable it and restrict the type of fields which can contribute to the condensates. The character of fields in $\mathcal{F}$ has to be defined self-consistently on the basis of quantum effective action. Enabling the gluon condensate means that gauge fields $A_{\mu}^{a}$ should satisfy

$\mathcal{F}=\left\{A: \lim _{V \rightarrow \infty} \frac{1}{V} \int_{V} d^{4} x g^{2} F_{\mu \nu}^{a}(x) F_{\mu \nu}^{a}(x)=B^{2}\right\}$.

First of all, the requirement of nonzero condensate $B^{2} \neq 0$ singles out fields $B_{\mu}^{a}$ with the strength which is constant almost everywhere in $R^{4}$, i.e. the part of $R^{4}$ where the field is inhomogenous has measure (4-volume) zero. The rest of deviations from homogeneity can be treated as fluctuations in the background of $B_{\mu}^{a}$. Separation of the long range modes $B_{\mu}^{a}$ responsible for gluon condensate and the local fluctuations $Q_{\mu}^{a}$ in the background $B_{\mu}^{a}$, must be supplemented by the gauge fixing condition. The background gauge condition $D(B) Q=0$ is the most natural choice. At the formal level, the separation can 
be achieved by the insertion of identity

$$
\begin{gathered}
1=\int_{\mathcal{B}} D B \Phi[A, B] \int_{\mathcal{Q}} D Q \int_{\Omega} D \omega \delta\left[A^{\omega}-Q^{\omega}-B^{\omega}\right] \\
\times \delta\left[D\left(B^{\omega}\right) Q^{\omega}\right], \\
A_{\mu}^{a}=B_{\mu}^{a}+Q_{\mu}^{a},
\end{gathered}
$$

where $Q$ are fluctuations of the gluon field with zero gluon condensate: $Q \in \mathcal{Q}$. Field $B_{\mu}^{a}$ are long range field configurations with, in general, the nonzero condensate: $B \in \mathcal{B}$. Performing the standard Faddeev-Popov procedure one arrives at

$$
\begin{aligned}
Z= & N^{\prime} \int_{\mathcal{B}} D B \int_{\mathcal{Q}} D Q \operatorname{det}[D(B) D(B+Q)] \\
& \times \delta[D(B) Q] \exp \{-S[B+Q]\}
\end{aligned}
$$

The character of long-range fields has yet to be identified by the dynamics of fluctuations $Q$. At the formal level, integral over $Q$ defines an effective action for the long range part of the gluon field

$$
Z=N^{\prime} \int_{\mathcal{B}} D B \exp \left\{-S_{\text {eff }}[B]\right\}
$$

Gluon fields $B_{\mu}^{a}$, which correspond to the global minima of $S_{\text {eff }}[B]$, dominate over the integral in the thermodynamic limit $V \rightarrow \infty$ and define the phase structure of the system. First of all, one has to take a look at fields with just constant strength. There are two different kinds of this type of fields: Abelian covariantly constant fields $B_{\mu}^{a}=-\frac{1}{2} n^{a} B_{\mu \nu} x_{\nu}$ and non-Abelian constant vector potentials $B_{\mu}^{a}=$ const. Unlike the former, non-Abelian fields are unstable against small perturbations $Q_{\mu}^{a}$ (for comprehensive discussion of the effective potential in pure YangMills theory see 3, 44. Pagels and Tomboulis studied an effective action for these fields within the context of scale anomaly [5], Woloshyn and Trottier attempted lattice calculation [6]. All these calculations indicated a minimum of the effective action at nonzero Abelian (anti-)self-dual field. Recently, the effective potential was calculated within the functional RG [7]. The result has also indicated a minimum of the effective action at the nonzero Abelian (anti-)self-dual field. In [8], the Landau-Ginsburg Lagrangian for pure Yang-Mills gauge fields invariant under the standard space-time and local gauge $S U(3)$ transformations was considered. It has been demonstrated that for $N_{\mathrm{c}}=3$ a set of twelve degenerated minima of the action density exists as soon as a nonzero gluon condensate is postulated in the action. The minima are connected to each other by the Weyl group transformations associated with the color su(3) algebra and parity transformation. The presence of degenerated discrete minima in the Lagrangian leads to the solutions of the effective equations of motion in the form of the kink-like gauge field configurations interpolating between different minima. The homogeneous field with a kink defect is the simplest example of gluon configurations which are homogeneous almost everywhere in $R^{4}$ and satisfy the basic condition Eq.(11). The spectrum of covariant derivative squared $D^{2}$ in the presence of the simplest solution, which interpolates between selfdual and anti-self-dual Abelian homogeneous fields, was estimated. This kink configuration can be seen as a domain wall defect separating the regions with self-dual and anti-self-dual Abelian gauge field. On the domain wall the gluon field is Abelian with orthogonal to each other chromomagnetic and chromoelectric fields. For the aims of the present study it is important that the spectrum of $D^{2}$ or $D$ in the (anti-)self-dual field is purely discrete with bound state type eigenfunctions while for the crossed orthogonal fields the spectrum is continuous with the Landau level structure and the corresponding wave eigen functions.

The eigenvalues and the square integrable eigenfunctions of $D^{2}$ for the (anti-)self-dual field are

$$
\begin{aligned}
\lambda_{r} & =4 B(r+1) \\
\phi_{n m k l}(x) & =C_{n m k l}\left(\beta_{+}^{+}\right)^{k}\left(\beta_{-}^{+}\right)^{l}\left(\gamma_{+}^{+}\right)^{n}\left(\gamma_{-}^{+}\right)^{m} \phi_{0}(x), \\
\phi_{0}(x) & =e^{-\frac{1}{2} B x^{2}}, C_{n m k l}=\frac{1}{\sqrt{n ! m ! k ! l !} \pi^{2}},
\end{aligned}
$$

where $r=k+n$ for the self-dual field, $r=l+n$ for the anti-self-dual field, $\beta_{ \pm}^{ \pm}$and $\gamma_{ \pm}^{ \pm}$are related to a set of creation and annihilation operators (details can be found in [8]). The spectrum is discrete. In this background no color charged waves are enabled, and there are no charged particle degrees of freedom. This is understood below as confinement of dynamical charged fields.

Inside the infinitely thin domain wall placed at $x_{1}=0$ with the chromomagnetic field directed along the $y$ axis and the chromoelectric field along the $z$ axis the charged scalar field displays a continuous spectrum similar to the Landau levels. The eigen functions square integrable over $x_{3}$ take the form

$\phi_{n}\left(p_{2}, p_{4} \mid x_{2}, x_{3}, x_{4}\right)=\exp \left(-i p_{4} x_{4}-i p_{2} x_{2}\right) \chi_{n}\left(p_{4} \mid x_{3}\right)$,

where the functions $\chi_{n}$ are

$$
\begin{aligned}
\chi_{n}\left(p_{4} \mid x_{3}\right)= & \exp \left\{-2 \sqrt{2} B\left(x_{3}+\frac{p_{4}}{4 B}\right)^{2}\right\} \\
& \times H_{n}\left(2^{3 / 4} \sqrt{B}\left(x_{3}+\frac{p_{4}}{4 B}\right)\right) .
\end{aligned}
$$


The eigenvalues look like

$$
\lambda_{n}\left(p_{2}^{2}, p_{4}^{2}\right)=2 \sqrt{2} B(2 n+1)+p_{2}^{2}+p_{4}^{2},
$$

and correspond to the color charged quasiparticles with mass $m_{n}^{2}=2 \sqrt{2} B(2 n+1)$ freely moving along the chromomagnetic field:

$$
p_{0}^{2}=p_{2}^{2}+m_{n}^{2}
$$

The purely discrete spectrum and bound state (four-dimentional oscillator) eigen functions can be treated as confinement of color charged fields in the (anti-)self-dual homogeneous field (in the bulk of $\left.R^{4}\right)$. Landau levels and wave eigenfunctions indicate the absence of confinement at the domain wall. In other words, charged particles are localized at the wall.

It should be noted here that accurate separation of the specific Abelian part $B$ of general vector potential $A$ as in Eq.(2) is a complicated problem. The methods to tackle the problem were studied in [10, 12 -15].

For completeness, we have to mention that in the context of center symmetry the dominance of the lumpy gauge field configurations was discussed in lattice calculations [16 20]. In paper [18], an effective model of $S U(2)$ gauge theory for the domain wall formation was considered.

The idea of the dominance of the gluon fields which are (anti-)self-dual Abelian almost everywhere turned out to be phenomenologically efficient. The model of confinement, chiral symmetry breaking and hadronization based on the ensemble of Abelian (anti-)self-dual fields was developed in a series of papers 21 23]. In the model, the direction of the gauge field in space and color space, and the duality of the field are random parameters of the domains as well as pisitions of domain centers. All configurations of this type are summed up in the partition function. The domain model exhibits confinement of static (square law) and dynamical quarks (absence of poles in the propagators of color charged fields, discrete spectrum of the corresponding differential operator), spontaneous breaking of the flavour chiral symmetry, $U_{\mathrm{A}}(1)$ symmetry is broken due to the axial anomaly, strong $C P$ violation is absent in the model. With a minimal set of parameters (meson masses, gauge coupling constant, gluon condensate and mean domain size) the model gives rather accurate results for meson masses from all different parts of the spectrum: light mesons including excited states, heavy-light mesons, heavy quarkonia). The decay constants and some form factors were also calculated within the model. The above mentioned kink configurations have not been yet incorporated into the domain model directly but strongly motivate it.

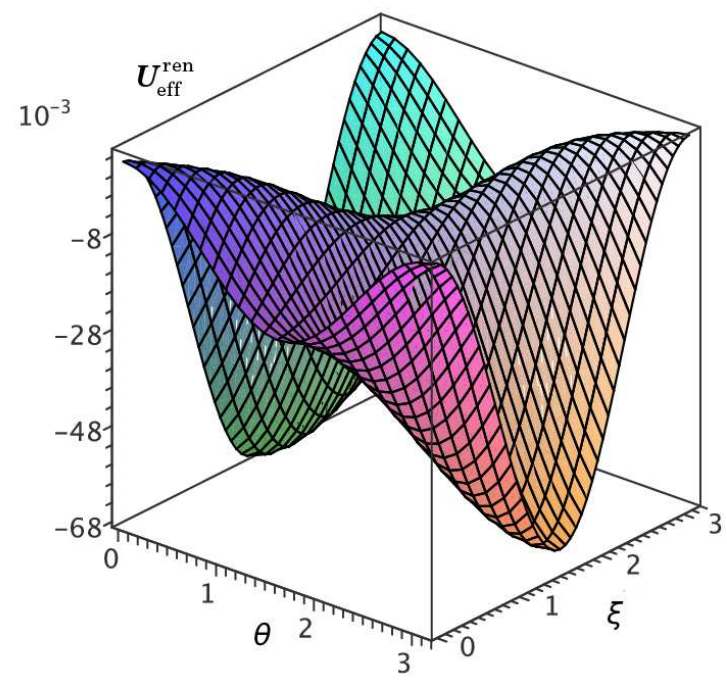

FIG. 1: Effective potential (in units of $B^{2} / 8 \pi^{2}$ ) as a function of angles $\theta$ and $\xi$ for the pure magnetic field $H=.9 B$ and $\phi=\chi$. The minimum is at $\theta=0, \xi=\pi / 2$.

Strong electromagnetic fields can emerge in relativistic heavy ion collisions 24 26]. Interplay of strong electromagnetic fields and nonperturbative gluon fields are expected to be important for understanding the dynamics of hadronic matter in heavy ion collisions. In particular, these fields can initiate such phenomena as chiral and vortic magnetic effects 24, 27, 28].

In this paper, we study an impact of the strong electromagnetic field on strong interactions in the context of lumpy or domain structured gluon fields. The one-loop quark contribution to the QCD effective potential for the homogeneous Abelian gluon fields in the presence of homogeneous electromagnetic field is evaluated. Extrema of the potential as a function of angles between chromoelectric, chromomagnetic and crossed orthogonal electromagnetic fields are analysed. In this setup the electromagnetic field is considered as an external one while the Abelian part of the gluon field represents domain structured nonperturbative gluon configurations related to QCD in the confinement phase. It is shown that the quark contribution is minimal for the crossed chromoelectric and chromomagnetic fields orthogonal to each other, which can be treated as a catalyzing impact of strong electromagnetic fields on deconfinement in hadronic matter. It should be stressed that this result has a very indirect relation to the real physics of heavy ion collision since it does not take into account the temperature and density effect. The present extremely simplified calculation can play an instructive role for more realistic con- 
sideration. The main qualitative result of this paper

could trigger a deconfinement transition in QCD.

is an observation that strong electromagnetic field

\section{ONE-LOOP QUARK CONTRIBUTION TO THE EFFECTIVE POTENTIAL IN THE PRESENCE OF ARBITRARY HOMOGENOUS ABELIAN FIELDS}

The one-loop contribution to the QCD effective potential is defined by the Gaussian integral over quark fields $\psi$ with the covariant derivative which includes both the electromagnetic field and the Abelian homogeneous gluon field

$$
\begin{aligned}
& e^{-V U_{\mathrm{eff}}(G)}=\mathcal{N} \int D \psi D \bar{\psi} e^{\int d^{4} x \bar{\psi}(x)(i \not D-m) \psi(x)}, \\
& U_{\mathrm{eff}}(G)=-\frac{1}{V} \ln \frac{\operatorname{det}(i \not D-m)}{\operatorname{det}(i \partial \partial-m)} \\
& =\frac{1}{V} \int_{V} d^{4} x \operatorname{Tr} \int_{m}^{\infty} d m^{\prime}\left[S\left(x, x \mid m^{\prime}\right)-S_{0}\left(x, x \mid m^{\prime}\right)\right]
\end{aligned}
$$

where $S(x, y \mid m)$ is the fermion propagator in external gauge fields with mass $m$. The following notation is used

$$
\begin{aligned}
& \not D=\gamma_{\mu} D_{\mu}, \quad D_{\mu}=\partial_{\mu}-i G_{\mu}, \quad G_{\mu}=\hat{B}_{\mu}+q A_{\mu} \\
& G_{\mu}=-\frac{1}{2} G_{\mu \nu} x_{\nu}, \quad G_{\mu \nu}=q F_{\mu \nu}+\hat{n} B_{\mu \nu} \\
& G_{i j}=\varepsilon_{i j k} \mathcal{H}_{k}, \quad G_{4 k}=\mathcal{E}_{k}, \\
& \overrightarrow{\mathcal{H}}=\hat{n} \mathbf{H}_{\mathrm{gl}}+q \mathbf{H}, \quad \overrightarrow{\mathcal{E}}=\hat{n} \mathbf{E}_{\mathrm{gl}}+q \mathbf{E}
\end{aligned}
$$

Here electromagnetic fields are denoted as $\mathbf{H}$ and $\mathbf{E}$, and $\mathbf{H}_{\mathrm{gl}}$ and $\mathbf{E}_{\mathrm{gl}}-$ chromomagnetic and chromoelectric fields of the same value $\mathbf{H}_{\mathrm{gl}}=\mathbf{E}_{\mathrm{gl}}=B, q$ is quark electric charge. Trace includes sum of the elements of diagonal matrices $\hat{n}, m$ and $q$. Two invariants of the gauge fields are

$$
\mathcal{R}=\frac{1}{4} G_{\mu \nu} G_{\mu \nu}=\frac{1}{2}\left(\overrightarrow{\mathcal{H}}^{2}+\overrightarrow{\mathcal{E}}^{2}\right), \quad \mathcal{Q}=G_{\mu \nu} \tilde{G}_{\mu \nu}=\overrightarrow{\mathcal{H}} \overrightarrow{\mathcal{E}}
$$

The quark propagator can be calculated analytically (see appendix $\underline{\mathrm{A}}$ ),

$$
S(x, y \mid m)=\left(m+i \not D_{x}\right) H(x, y \mid m)
$$

with

$$
\begin{aligned}
& H(x, y \mid m)=\frac{1}{m^{2}+\not D^{2}} \delta(x-y)=e^{-\frac{i}{2} x_{\mu} G_{\mu \nu} y_{\nu}} \frac{\mathcal{Q}}{16 \pi^{2}} \int_{0}^{\infty} d s \frac{e^{-m^{2} s}}{\sinh \left(s \sqrt{\mathcal{Q} \sigma_{-}}\right) \sinh \left(s \sqrt{\mathcal{Q} \sigma_{+}}\right)} \\
& {\left[P_{+} \cosh (s|\overrightarrow{\mathcal{E}}-\overrightarrow{\mathcal{H}}|)+P_{-} \cosh (s|\overrightarrow{\mathcal{E}}+\overrightarrow{\mathcal{H}}|)-\frac{1}{2} \sigma_{\mu \nu}\left[G_{\mu \nu}-\tilde{G}_{\mu \nu}\right] \frac{\sinh (s|\overrightarrow{\mathcal{E}}-\overrightarrow{\mathcal{H}}|)}{|\overrightarrow{\mathcal{E}}-\overrightarrow{\mathcal{H}}|}-\frac{1}{2} \sigma_{\mu \nu}\left[G_{\mu \nu}+\tilde{G}_{\mu \nu}\right] \frac{\sinh (s|\overrightarrow{\mathcal{E}}+\mathcal{\mathcal { H }}|)}{|\overrightarrow{\mathcal{E}}+\overrightarrow{\mathcal{H}}|}\right]} \\
& \times \exp \left\{-\frac{\sqrt{\mathcal{Q} \sigma_{+}} \operatorname{coth}\left(s \sqrt{\mathcal{Q} \sigma_{-}}\right)-\sqrt{\mathcal{Q} \sigma_{-}} \operatorname{coth}\left(s \sqrt{\mathcal{Q} \sigma_{+}}\right)}{4\left(\sigma_{+}-\sigma_{-}\right)}(x-y)^{2}\right. \\
& -\frac{\sqrt{\mathcal{Q} \sigma_{+}} \operatorname{coth}\left(s \sqrt{\mathcal{Q} \sigma_{+}}\right)-\sqrt{\mathcal{Q} \sigma_{-}} \operatorname{coth}\left(s \sqrt{\mathcal{Q} \sigma_{-}}\right)}{4 \mathcal{Q}\left(\sigma_{+}-\sigma_{-}\right)}(x) \\
& P_{ \pm}=\frac{1}{2}\left(1 \pm \gamma_{5}\right), \quad \sigma_{\mu \nu}=\frac{1}{2 i}\left[\gamma_{\mu}, \gamma_{\nu}\right], \quad \sigma_{ \pm}=\frac{\mathcal{R}}{\mathcal{Q}}\left(1 \pm \sqrt{1-\frac{\mathcal{Q}^{2}}{\mathcal{R}^{2}}}\right) .
\end{aligned}
$$


Using this propagator one gets for the effective potential

$$
U_{\text {eff }}^{\text {ren }}(G)=\operatorname{Tr} \frac{\mathcal{Q}}{8 \pi^{2}} \int_{s_{0}}^{\infty} \frac{d s}{s} e^{-m^{2} s} \frac{\cosh (s|\overrightarrow{\mathcal{E}}-\overrightarrow{\mathcal{H}}|)+\cosh (s|\overrightarrow{\mathcal{E}}+\overrightarrow{\mathcal{H}}|)}{\sinh \left(s \sqrt{\mathcal{Q} \sigma_{-}}\right) \sinh \left(s \sqrt{\mathcal{Q} \sigma_{+}}\right)} .
$$

Here $s_{0}$ regularises the UV divergence of the integral, trace denotes summation over the elements of the diagonal color matrix $\hat{n}$ as well as quark charges $q$ and masses $m$ for all flavours under consideration. Using the identities

$$
\begin{aligned}
& \cosh (s|\overrightarrow{\mathcal{E}} \pm \overrightarrow{\mathcal{H}}|)=\cosh \left(s\left(\rho_{+} \pm \rho_{-}\right)\right), \quad \sqrt{\mathcal{Q} \sigma_{ \pm}}=\rho_{ \pm} \\
& \rho_{ \pm}=\frac{1}{\sqrt{2}}(\sqrt{\mathcal{R}+\mathcal{Q}} \pm \sqrt{\mathcal{R}-\mathcal{Q}})
\end{aligned}
$$

one arrives at the renormalized effective potential written in terms of invariants $\mathcal{R}$ and $\mathcal{Q}$

$$
\begin{gathered}
U_{\mathrm{eff}}(G)=U_{\mathrm{eff}}^{\mathrm{ren}}(G)+\delta U_{\mathrm{eff}}, \quad \delta U_{\mathrm{eff}}=\frac{1}{8 \pi^{2}}\left(\operatorname{Tr} \frac{2}{3} \mathcal{R}\right) \int_{s_{0}}^{\infty} \frac{d s}{s} e^{-\frac{m^{2}}{B} s}, \\
U_{\mathrm{eff}}^{\mathrm{ren}}(G)=\frac{B^{2}}{8 \pi^{2}} \int_{0}^{\infty} \frac{d s}{s^{3}} \operatorname{Tr}_{n}\left[s \varkappa_{+} \operatorname{coth}\left(s \varkappa_{+}\right) s \varkappa_{-} \operatorname{coth}\left(s \varkappa_{-}\right)-1-\frac{s^{2}}{3}\left(\varkappa_{+}^{2}+\varkappa_{-}^{2}\right)\right] e^{-\frac{m^{2}}{B} s}, \\
\varkappa_{ \pm}=\frac{1}{\sqrt{2} B}(\sqrt{\mathcal{R}+\mathcal{Q}} \pm \sqrt{\mathcal{R}-\mathcal{Q}}) .
\end{gathered}
$$

Through the identity (6) this expression can be reduced to the well known form of the effective potential, see [11] and references therein.

For the experimantal situation of heavy ion collisions, which we bear in mind, it is sufficient to consider the electric and magnetic fields orthogonal to each other and choose coordinate system with the $z$-axis along the magnetic field and the $x$-axis along the electric field,

$$
H_{i}=\delta_{i 3} H, \quad E_{i}=\delta_{i 1} E, \quad \mathbf{E H}=0,
$$

For the physical electric field orthogonal to the magnetic field invariants $\mathcal{R}$ and $\mathcal{Q}$ read

$$
\begin{aligned}
& \mathcal{R}=\left(H^{2}-E^{2}\right) / 2+\hat{n}^{2} B^{2}+\hat{n} B H \cos (\theta)+i B E \cos (\chi) \sin (\xi), \\
& \mathcal{Q}=\hat{n} B H \cos (\xi)+i \hat{n} B E \sin (\theta) \cos (\phi)+\hat{n}^{2} B^{2}(\sin (\theta) \sin (\xi) \cos (\phi-\chi)+\cos (\theta) \cos (\xi)),
\end{aligned}
$$

where $(\phi, \theta)$ are the spherical angles of the chromomagnetic field, and $(\chi, \xi)$ are the spherical angles of the chromoelectric field in the chosen coordinate system.

\section{MINIMA OF THE EFFECTIVE POTENTIAL}

For the pure magnetic field $(E=0)$ the renormalised effective potential is real. It depends on $\theta$, $\xi$ and the difference between $\phi$ and $\chi$. Figure 1 represents the effective potential for the case of three quark flavours, the masses are taken the same for all flavours. A minimum is achieved at $\theta=0, \xi=\pi / 2$, i.e. for the crossed orthogonal chromomagnetic and chromoelectric fields. The value of the potential does not depend on $\phi$ and $\chi$. In other words, the chromomagnetic field is collinear to the magnetic field, and the chromoelectric field is orthogonal to the chromomagnetic field. The polar angle $\chi$ of $\mathbf{E}_{\mathrm{gl}}$ is not fixed.

If the electric field is nonzero, the effective potential becomes comlex. The analytical properties of the effective potential as a function of complex $\mathcal{Q}$ and $\mathcal{R}$ can be studied by means of the convergent series representation of the integral (77) obtained in [11]. The nonzero imaginary part of the potential would mean instability in the system. However, the imaginary part does vanish at the pont in the space of angles where the real part is minimal. This can be verified by inspection through the straightforward 
calculation of the real and imaginary parts of the potential. The simplest way to find this stable minimum of the potential is to require that the RHS of Eqs. (8) for $\mathcal{R}$ and $\mathcal{Q}$ to be real, which can be achieved by restriction imposed on the angles

$$
\begin{gathered}
\sin (\xi) \cos (\chi)=0 \\
\sin (\theta) \cos (\phi)=0 .
\end{gathered}
$$

Figures 2 and 3 illustrate that a globall minimum is achieved at $\theta=0, \xi=\pi / 2, \chi=\pi / 2$ or $3 \pi / 2$. It does not depend on $\phi$. This minimum corresponds to the crossed orthogonal to each other chromoelectric and chromomagnetic fields, the chromomagnetic field is collinear to the magnetic field, and the chromoelectric field is orthogonal to both magnetic and electric fields. The minimum exists for any value of the electromagnetic fields. A value of the potential at the minimum depends on their strengh.

\section{DISCUSSION.}

We have analysed dependence of the one-loop quark contribution to the QCD effective action on the angles between physical homogeneous electromagnetic field and Abelian homogeneous Euclidean gluon field. The main result consists in observation that in the presence of the external orthogonal magnetic and electric fields there exists a global mimium corresponding to the crossed orthogonal chromomagnetic and chromoelectric fields. Unlike the (anti-)self-dual homogeneous gluon field minimising the effective potential in pure gluodynamics [1, 4, 5, 7], the crossed orthogonal gluon field does not support confinement of quarks: the color charged quasi-particles do exist and can move along the direction of the magnetic field, see Eq.(3) . In this sense, strong electromagnetic field can rearrange the structure of the global minima of the effective action of QCD.

If to take a liberty to extrapolate this extremely simplified setup to the situation of relativistic heavy ion collision, then one can expect that a strong electromagnetic field generated during the collision triggers quark deconfinement transition in hadronic matter. Within the context of the domain model and the kink solution one can think that a strong electromagnetic field produces a domain wall defect in the confining gluon background exactly in the region where collision occurs. If so then deconfined quarks will move preferably along the direction of magnetic field but this will happen due to the gluon field configuration, due to QCD interaction, even after the switching the electromagnetic field off. However, prior to discussing the phenomenologically relevant observables arising from this effect, we should make the computation more realistic. First of all,
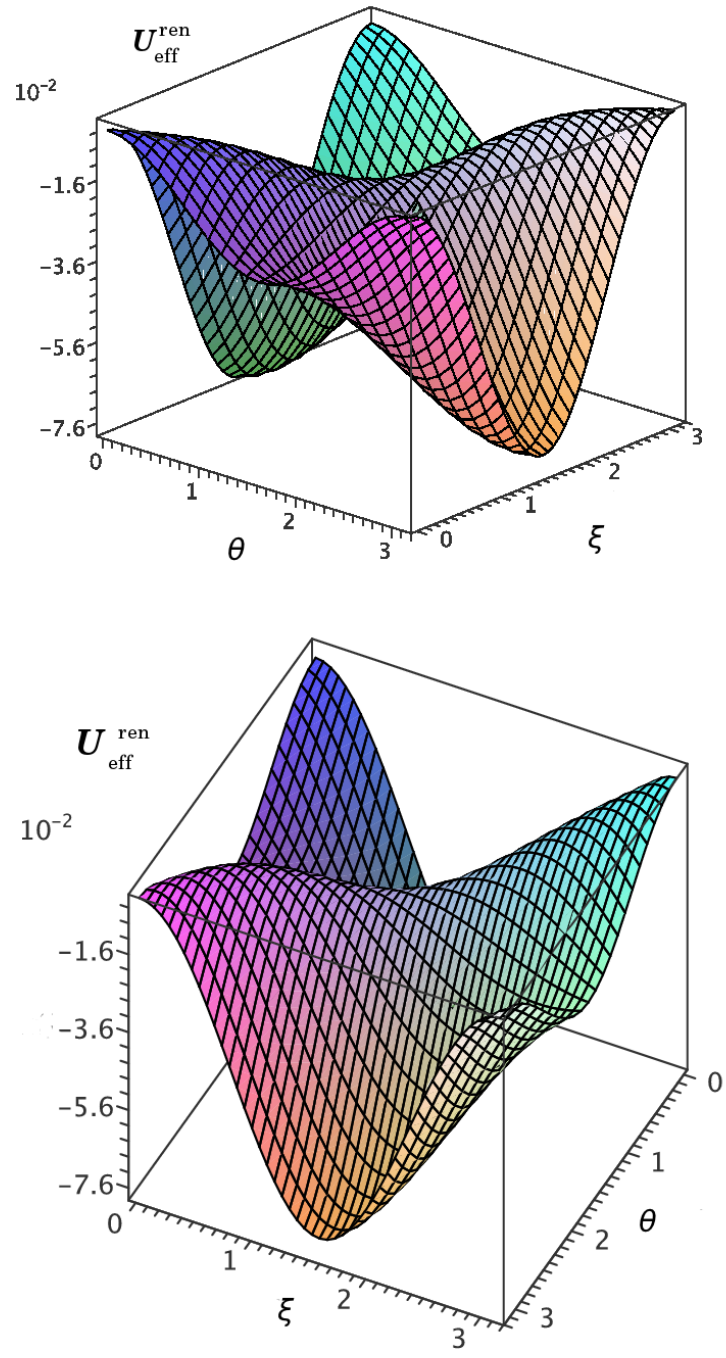

FIG. 2: Effective potential (in units of $B^{2} / 8 \pi^{2}$ ) for the electric $E=.5 B$ and the magnetic $H=.9 B$ fields as a function of the angles $\theta$ and $\xi(\phi=\chi=\pi / 2$ - upper figure, $\phi=\pi / 2, \chi=3 \pi / 2$ - lower figure ).

this would imply incorporation of the temperature and baryonic density effects, and taking an inhomogeneity of the gauge fields into account as far as possible.

\section{ACKNOWLEDGEMENTS}

We acknowledge fruitful discussions with Vyacheslav Toneev and Dmitri Pak. 


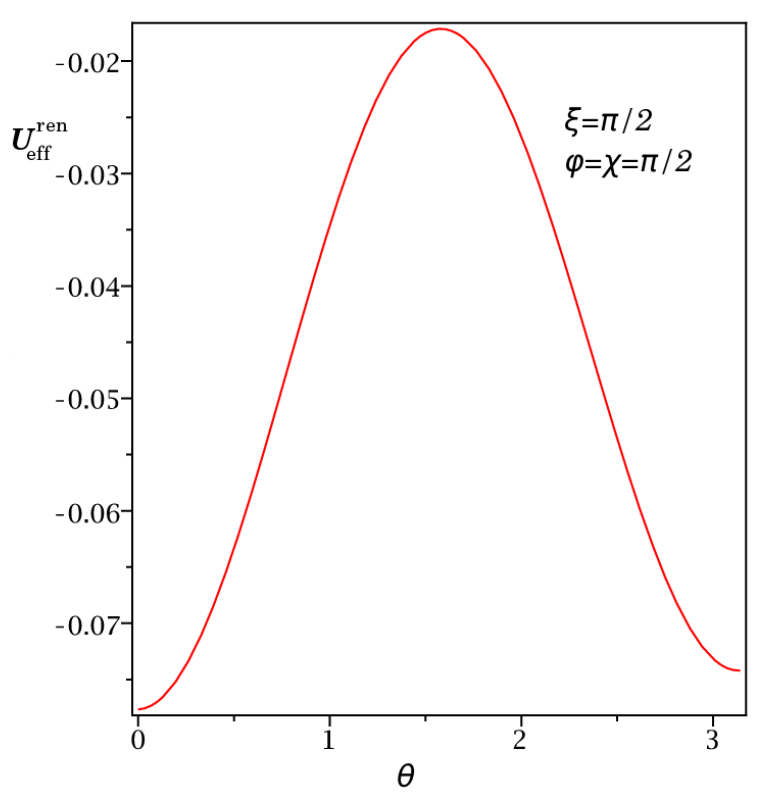

FIG. 3: Effective potential (in units of $B^{2} / 8 \pi^{2}$ ) for the electric $E=.5 B$ and the magnetic $H=.9 B$ fields as a function of the angle $\theta$ and $\xi=\pi / 2(\phi=\chi=\pi / 2)$.

\section{Appendix A: Quark propagator in the presence} of arbitrary homogenous Abelian fields

The quark propagator

$$
\begin{aligned}
& S(x, y \mid m)=\frac{1}{m-i \not D_{x}} \delta(x-y)=\left(m+i \not D_{x}\right) H(x, y \mid m), \\
& H(x, y \mid m)=\frac{1}{m^{2}+\not D^{2}} \delta(x-y),
\end{aligned}
$$

can be represented in the form

$$
H(x, y)=\int_{0}^{\infty} d s e^{-m^{2} s} e^{-\frac{1}{2} s(\sigma G)} e^{s D^{2}} \delta(x-y)
$$

with

$$
\begin{aligned}
\not D^{2} & =-D^{2}+\frac{1}{2} G_{\mu \nu} \sigma_{\mu \nu} \\
(\sigma G) & =\sigma_{\mu \nu} G_{\mu \nu}, \quad \sigma_{\mu \nu}=\frac{1}{2 i}\left[\gamma_{\mu}, \gamma_{\nu}\right] .
\end{aligned}
$$

Using relations

$$
\begin{aligned}
& \left(\frac{1}{2}(\sigma G)\right)^{2 n}=(\mathcal{E}-\mathcal{H})^{2 n} P_{+}+(\mathcal{E}+\mathcal{H})^{2 n} P_{-}, \\
& (\sigma G) P_{ \pm}=\frac{1}{2} \sigma_{\mu \nu}\left[G_{\mu \nu} \mp \tilde{G}_{\mu \nu}\right] \\
& P_{ \pm}=\frac{1}{2}\left(1 \pm \gamma_{5}\right), \tilde{G}_{\mu \nu}=\frac{1}{2} \varepsilon_{\mu \nu \alpha \beta} G_{\alpha \beta}
\end{aligned}
$$

one can get

$$
\begin{aligned}
e^{-\frac{1}{2} s(\sigma G)} & =P_{+} \cosh (s|\overrightarrow{\mathcal{E}}-\overrightarrow{\mathcal{H}}|)+P_{-} \cosh (s|\overrightarrow{\mathcal{E}}+\overrightarrow{\mathcal{H}}|) \\
& -\frac{1}{2} \sigma_{\mu \nu}\left[G_{\mu \nu}-\tilde{G}_{\mu \nu}\right] \frac{\sinh (s|\overrightarrow{\mathcal{E}}-\overrightarrow{\mathcal{H}}|)}{|\overrightarrow{\mathcal{E}}-\overrightarrow{\mathcal{H}}|} \\
& -\frac{1}{2} \sigma_{\mu \nu}\left[G_{\mu \nu}+\tilde{G}_{\mu \nu}\right] \frac{\sinh (s|\overrightarrow{\mathcal{E}}+\overrightarrow{\mathcal{H}}|)}{|\overrightarrow{\mathcal{E}}+\overrightarrow{\mathcal{H}}|} . \quad(\mathrm{A} 2)
\end{aligned}
$$

The integrand in Eq. (44) can be computed by means of the path integral representation,

$$
\begin{aligned}
& \exp \left\{s D^{2}\right\} \delta(x-y)=\int \delta a_{\mu} P_{\beta} \exp \left\{-\int_{0}^{1} d \beta a^{2}(\beta)\right. \\
& \left.+2 \sqrt{s} \int_{0}^{1} d \beta a_{\mu}(\beta) D_{\mu}\right\} \delta(x-y) \\
& =\frac{1}{16 \pi^{2} s^{2}} \exp \left\{-\frac{(x-y)^{2}}{4 s}-\frac{i}{2} x_{\mu} G_{\mu \nu} y_{\nu}\right\} \prod_{k=1}^{\infty} \sqrt{\operatorname{det} O(k)} \\
& \quad \times \exp \left\{\sum_{n=1}^{\infty}-\frac{2 s}{\pi^{2} n^{2}} G_{\mu}(x-y) O_{\mu \nu}^{-1}(n) G_{\nu}(x-y)\right\},
\end{aligned}
$$

where

$$
O_{\nu \alpha}(n)=\left[\delta_{\nu \alpha}+\frac{s^{2}}{\pi^{2} n^{2}} G_{\mu \nu} G_{\mu \alpha}\right],
$$

and the determinant is

$$
\begin{aligned}
& \operatorname{det} O(k)=\operatorname{det}\left[I-\frac{s^{2}}{\pi^{2} n^{2}} G_{\mu \rho} G_{\rho \nu}\right] \\
& =\left[1+\frac{(\overrightarrow{\mathcal{E}} \overrightarrow{\mathcal{H}})^{2} s^{4}}{\pi^{4} k^{4}}+\frac{\left(\overrightarrow{\mathcal{E}}^{2}+\overrightarrow{\mathcal{H}}^{2}\right) s^{2}}{\pi^{2} k^{2}}\right]^{2} .
\end{aligned}
$$

With the notation

$$
\begin{aligned}
& \mathcal{Q}=(\mathcal{E} \mathcal{H}), \quad \mathcal{R}=\frac{1}{2}\left(\mathcal{E}^{2}+\mathcal{H}^{2}\right), \\
& \sigma_{ \pm}=\frac{\mathcal{R}}{\mathcal{Q}}\left(1 \pm \sqrt{1-\frac{\mathcal{Q}^{2}}{\mathcal{R}^{2}}}\right), \quad \sigma_{+} \sigma_{-}=1
\end{aligned}
$$

one arrives at

$$
\begin{aligned}
\operatorname{det} O(k) & =\left\{\left[1+\frac{\mathcal{Q} s^{2} \sigma_{-}}{\pi^{2} k^{2}}\right]\left[1+\frac{\mathcal{Q} s^{2} \sigma_{+}}{\pi^{2} k^{2}}\right]\right\}^{2}, \\
{\left[\prod_{k=0}^{\infty} \operatorname{det} O^{1 / 2}(k)\right]^{-1} } & =\frac{s^{2} \mathcal{Q}}{\sinh \left(s \sqrt{\mathcal{Q} \sigma_{-}}\right) \sinh \left(s \sqrt{\mathcal{Q} \sigma_{+}}\right)} .
\end{aligned}
$$


The sum in the exponent with $O_{\mu \nu}^{-1}$ can be evaluated as

$$
\sum_{n=1}^{\infty} \frac{2 s}{\pi^{2} n^{2}} G_{\mu}(x-y) O_{\mu \nu}^{-1}(n) G_{\nu}(x-y)=G_{\mu}(x-y) G_{\mu}(x-y) \Sigma_{1}+(x-y)^{2} \Sigma_{2},
$$

where the terms $\Sigma_{1}$ and $\Sigma_{2}$ are given by

$$
\begin{aligned}
\Sigma_{1} & =\sum_{n=1}^{\infty} \frac{2 s \pi^{2} n^{2}}{\pi^{4} n^{4}+2 s^{2} \pi^{2} n^{2} \mathcal{R}+\mathcal{Q}^{2} s^{4}}=\frac{2 \sigma_{-} s}{\left(\sigma_{-}-\sigma_{+}\right)} \sum_{n=1}^{\infty} \frac{1}{\pi^{2} n^{2}+s^{2} \mathcal{Q} \sigma_{-}}+\frac{2 \sigma_{+} s}{\left(\sigma_{+}-\sigma_{-}\right)} \sum_{n=1}^{\infty} \frac{1}{\pi^{2} n^{2}+s^{2} \mathcal{Q} \sigma_{+}} \\
& =\frac{\sqrt{\mathcal{Q} \sigma_{+}} \operatorname{coth}\left(s \sqrt{\mathcal{Q} \sigma_{+}}\right)-\sqrt{\mathcal{Q} \sigma_{-}} \operatorname{coth}\left(s \sqrt{\mathcal{Q} \sigma_{-}}\right)}{\left.\Sigma_{2}-\sigma_{-}\right)} \\
\Sigma_{2} & =\frac{1}{2} \sum_{n=1}^{\infty} \frac{s^{3} \mathcal{Q}^{2}}{\pi^{4} n^{4}+2 s^{2} \pi^{2} n^{2} \mathcal{R}+\mathcal{Q}^{2} s^{4}}=\frac{s \mathcal{Q}}{2\left(\sigma_{+}-\sigma_{-}\right)} \sum_{n=1}^{\infty}\left[\frac{1}{\pi^{2} n^{2}+\frac{s^{2} \mathcal{Q}}{\sigma_{+}}}-\frac{1}{\pi^{2} n^{2}+\frac{s^{2} \mathcal{Q}}{\sigma_{-}}}\right] \\
& =\frac{\sqrt{\mathcal{Q} \sigma_{+}} \operatorname{coth}\left(s \sqrt{\mathcal{Q} \sigma_{-}}\right)-\sqrt{\mathcal{Q} \sigma_{-}} \operatorname{coth}\left(s \sqrt{\mathcal{Q} \sigma_{+}}\right)}{4\left(\sigma_{+}-\sigma_{-}\right)}-\frac{1}{4 s}
\end{aligned}
$$

as a result of this calculation one gets representation

$$
\begin{aligned}
& \exp \left\{s D^{2}\right\} \delta(x-y)=\frac{1}{16 \pi^{2}} \frac{\mathcal{Q}}{\sinh \left(s \sqrt{\mathcal{Q} \sigma_{-}}\right) \sinh \left(s \sqrt{\mathcal{Q} \sigma_{+}}\right)} \exp \left\{-\frac{i}{2} x_{\mu} G_{\mu \nu} y_{\nu}\right\} \\
& \times \exp \left\{-\frac{\sqrt{\mathcal{Q} \sigma_{+}} \operatorname{coth}\left(s \sqrt{\mathcal{Q} \sigma_{-}}\right)-\sqrt{\mathcal{Q} \sigma_{-}} \operatorname{coth}\left(s \sqrt{\mathcal{Q} \sigma_{+}}\right)}{4\left(\sigma_{+}-\sigma_{-}\right)}(x-y)^{2}\right. \\
& \left.-\frac{\sqrt{\mathcal{Q} \sigma_{+}} \operatorname{coth}\left(s \sqrt{\mathcal{Q} \sigma_{+}}\right)-\sqrt{\mathcal{Q} \sigma_{-}} \operatorname{coth}\left(s \sqrt{\mathcal{Q} \sigma_{-}}\right)}{4 \mathcal{Q}\left(\sigma_{+}-\sigma_{-}\right)} G_{\mu \nu} G_{\mu \rho}(x-y)_{\nu}(x-y)_{\rho}\right\} .
\end{aligned}
$$

Substitution of Eqs. (A2) and (A3) to Eq. (A1) leads to the quark propagator (44).

[1] P. Minkowski, Nucl. Phys. B177 (1981) 203.

[2] L. D. Faddeev, arXiv:0911.1013 [math-ph]].

[3] P. Minkowski, Phys. Lett. B 76 (1978) 439.

[4] H. Leutwyler, Phys. Lett. B96 (1980) 154; ibid Nucl. Phys. B 179 (1981) 129.

[5] H. Pagels, and E. Tomboulis, Nucl. Phys. B 143 (1978) 485.

[6] H. D. Trottier and R. M. Woloshyn, Phys. Rev. Lett. 70 (1993) 2053.

[7] A. Eichhorn, H. Gies and J. M. Pawlowski, Phys. Rev. D 83, 045014 (2011) [Erratum-ibid. D 83, 069903 (2011)] [arXiv:1010.2153 [hep-ph]].

[8] B.V. Galilo and S.N. Nedelko, Phys. Part. Nucl. Lett., 8 (2011) 67 [arXiv:hep-ph/1006.0248v2].

[9] Y.M. Cho Phys. Rev. Lett. 44 (1980) 1115.

[10] Y. M. Cho, J. H. Kim and D. G. Pak, Mod. Phys. Lett. A 21 (2006) 2789.

[11] Y.M. Cho, and D.G. Pak, Phys. Rev. Lett. 86 (2001) 1947.

[12] S.V. Shabanov, J. Math. Phys. 43 (2002) 4127 hep-th/0202146.

[13] S. V. Shabanov, Phys. Rept. 326 (2000) 1 arXiv:hep-th/0002043; S. V. Shabanov and
J. R. Klauder, Phys. Lett. B 456 (1999) 38. L. V. Prokhorov Yad. Fiz., 35 (1982) 229.

[14] L. D. Faddeev, A. J. Niemi, Nucl. Phys. B 776 (2007); ibid, Phys. Lett. B 449 (1999) 214.

[15] Kei-Ichi Kondo, Toru Shinohara, Takeharu Murakami, Prog. Theor. Phys. 120 (2008) 1 arXiv:0803.0176 [hep-th]].

[16] P. J. Moran and D. B. Leinweber arXiv:0805.4246 [hep-lat].

[17] P. J. Moran and D. B. Leinweber PoS LAT2007 (2007) 383 arXiv:0710.2380 [hep-lat]].

[18] Ph. de Forcrand, A. Kurkela and A. Vuorinen Phys. Rev. D 77 (2008) 125014.

[19] P. de Forcrand AIP Conf. Proc. 892 (2007) 29 arXiv:hep-lat/0611034.

[20] E. M. Ilgenfritz, et al, PoS LAT2007 (2007) 311 arXiv:0710.2607 [hep-lat]].

[21] G.V. Efimov, and S.N. Nedelko, Phys. Rev. D 51 (1995) 176; Ya. V. Burdanov, et. al, Phys. Rev. D 54 (1996) 4483.

[22] A.C. Kalloniatis and S.N. Nedelko, Phys. Rev. D 64 (2001) 114025;

[23] A.C. Kalloniatis and S.N. Nedelko, Phys. Rev. D 66 
(2002) 074020; ibid, Phys. Rev. D 69 (2004) 074029; Erratum-ibid. Phys. Rev. D 70 (2004) 119903; ibid, Phys. Rev. D 71 (2005) 054002; ibid, Phys. Rev. D 73 (2006) 034006.

[24] D. E. Kharzeev, L. D. McLerran, and H. J. Warringa, Nucl. Phys. A 803227 (2008)

[25] V. Voronyuk, V. D. Toneev, W. Cassing, E. L. Bratkovskaya, V. P. Konchakovski and S. A. Voloshin, arXiv:1103.4239 [nucl-th].
[26] V. Skokov, A. Y. Illarionov and V. Toneev, Int. J. Mod. Phys. A 24 (2009) 5925 arXiv:0907.1396 [nucl-th]].

[27] D. E. Kharzeev and D. T. Son, Phys. Rev. Lett. 106, 062301 (2011) arXiv:1010.0038 [hep-ph]].

[28] O. Rogachevsky, A. Sorin and O. Teryaev, Phys. Rev. C 82, 054910 (2010) arXiv:1006.1331 [hep$\mathrm{ph}]$. 